\title{
Editorial
}

\section{Not enough exercise?}

Once a year all researchers should read a few papers in their field written at least 50 years ago. In physiology at least they will be left with mixed feelings - dismay that they cannot approach the imagination, intelligence, and ingenuity of the giants of the past and relief that the same giants did not have access to modern technology. In no field are these reactions more justified than in exercise physiology, particularly when applied to assessment of functional reserve in the heart and lungs. Lewis, ${ }^{1}$ Barcroft, ${ }^{2}$ Douglas, ${ }^{3}$ and Haldane ${ }^{4}$ all understood the mechanisms influencing oxygen delivery and carbon dioxide removal and the importance of testing them under the load imposed by exercise. Since their time many studies have amply confirmed the value of measurement in exercise, but we have yet to see it used widely and routinely.

There are many clinical applications for exercise testing, which will not be reviewed in detail here. ${ }^{5}$ The information obtained may be helpful in quantifying and "explaining" exercise-related symptoms, such as fatigue, dyspnoea, wheezing, chest pain, and muscle pain; in revealing a diagnostic response, as in coronary artery disease; in suggesting an unexpected diagnosis, such as recurrent pulmonary emboli; in excluding a serious diagnosis, such as a cardiac murmur in a child; in quantifying the physiological reserve in the heart and lungs; and in the management of the patient through exercise prescription, occupational assessment, and evaluation of treatment.

In assessment of pulmonary disease simple measurements will show functional integration between respiratory drive, respiratory muscle power, and the mechanical characteristics of the total respiratory system, in terms of the tidal volume, the breathing frequency, and the timing of inspiration and expiration. Milic-Emilio has outlined an analytical scheme for respiratory control mechanisms which employs the respiratory duty cycle $\left(\mathrm{Ti} / \mathrm{T}_{\text {to }}\right.$ and the inspiratory occlusion pressure $(\mathrm{Pi})$, both measurements that may be made in exercise. Respiratory control mechanisms are reflected in the ventilatory responses to the increasing carbon dioxide production and through changes in alveolar or arterial carbon dioxide tension. ${ }^{8}$ Pulmonary gas exchange may be evaluated through measurements of dead space and venous admixture ${ }^{4} 10$ and by changes in arterial oxygen saturation, which may be measured indirectly by ear oximetry. ${ }^{11}$ Finally, the maximum power and oxygen intake are indices of integration between the links in the oxygen transport chain and as such reflect both the effects of a weak link and the ability to adapt to it.

Given this wealth of clinical information, why is it that exercise testing is not very widely used, except to identify the sagging ST segment? Perhaps the blame may be laid at the door of those who first used the phrase "stress test"; it sounds at the very least uncomfortable and potentially lethal. So long as the procedure is kept short, however, and the staff are well trained, an exercise test is safe and the shortlived discomfort well tolerated. In over 10000 tests we have encountered five serious problems-all ventricular arrhythmias in high-risk patients and all successfully managed. Perhaps it is due to a lack of communication between specialty groups - exercise testing may have become the victim of a demarcation dispute: cardiologists may not appreciate the benefit of or need for measurement of ventilation during exercise $^{12}$ and respiratory physiologists may tend to forget that the blood has to go through the lungs and that many pulmonary diseases are associated with impaired cardiac responses to exercise. ${ }^{13}$ Perhaps it is due to the apparent complexity of the studies, but complex measurements requiring sophisticated analysers, or arterial blood sampling, are not essential for most problems; neither is the "steady state," once thought so important by exercise physiologists. Or is it that the potential user, faced with so many published protocols and methods, concludes that exercise tests are more trouble than they are worth? At all events, hope may be near; there appears to be general agreement about many aspects of exercise testing, ${ }^{514-16}$ and several gatherings of experts have led to "recommendations."17-19

Firstly, there seems to be some agreement that an incremental exercise test is generally most useful and need not last longer than 10-15 minutes and that each increment in the work load need be maintained for only a minute, or even less. ${ }^{20}$ Secondly, so-called "submaximal tests" are uninformative and to be avoided except in a few well-defined circumstances, such as within a few weeks of myocardial infarction. They are no safer, ${ }^{21}$ and maximal oxygen intake and related variables cannot be estimated from submaximal measurements in patients with heart or lung disease. 1222 Thirdly, in most patients referred for 
exercise testing the necessary information may be obtained with simple, non-invasive measurements. More complex measurements, requiring gas analysis and arterial blood sampling, may then be reserved for those patients who need them. This means that the equipment required in an exercise laboratory may be kept simple and subsequently added to in the light of the type of patients referred.

A standardised test that has been widely used 15 can be carried out on a cycle ergometer or treadmill. 23 The exercise load is increased by a small amount each minute and at the end of each minute measurements are made of heart rate, ventilation, and the pattern of breathing. Measurements of blood pressure are also made frequently and the patient's symptoms are recorded by a sensory magnitude. ${ }^{24}$ The test is concluded because of fatigue or other symptoms experienced by the patient or because of the appearance of danger signs such as life-threatening arrhythmia or reduction in blood pressure. In appropriate patients 12-lead electrocardiographic records are obtained and the addition of ear oximetry is informative in respiratory patients.

The test will provide a measure of maximum exercise capacity, identify limiting factors, and show the evolution of cardiovascular and respiratory responses. In more than $90 \%$ of referred patients this information will be sufficient. In a few patients, however, more complex measurements of cardiac output and pulmonary gas exchange variables may be needed. In this case submaximal "steady-state" exercise levels may be chosen for study on the basis of the results of the progressive exercise test. 5 This allows the submaximal information to be interpreted in the light of the maximal study.

The observation of patients during exercise has always held promise; the proponents of the approach showed more than half a century ago that highpowered technology was not essential. Agreement on a standardised approach and an expansion of the clinical indications should lead to an increasing appreciation of its clinical value.

NL JONES

Ambrose Cardiorespiratory Unit, McMaster University Health Sciences Centre, Hamilton, Ontario,

Canada

\section{References}

${ }^{1}$ Lewis T. Diseases of the heart. London: MacMillan and Company, 1933.

2 Barcroft J. Features in the architecture of physiological function. London: Cambridge University Press, 1934.

${ }^{3}$ Douglas CG. Coordination of the respiration and circu- lation with variations in bodily activity. Lancet 1927;ii: 213.

${ }^{4}$ Haldane JS. Respiration. Princeton: Yale University Press, 1922.

5 Jones NL, Campbell EJM. Clinical exercise testing. 2nd ed. Philadelphia: Saunders Company, 1982.

6 Milic-Emili J. Recent advances in clinical assessment of control of breathing. Lung 1982;160:1-17.

${ }^{7}$ Sergysels R, Van Meerhaeghe A, Scano G, Denaut M, Willeput $R$. Respiratory drive during exercise in chronic obstructive lung disease. Bull Europ Physiopathol Respir 1981;17:755-66.

8 Jones NL. Use of exercise in testing respiratory control mechanisms. Chest 1976;70:171-3.

9 Jones NL, McHardy GJR, Naimark A, Campbell EJM. Physiological dead space and alveolar-arterial gas pressure differences during exercise. Clin Sci 1966;31: 19-29.

10 Jones NL. Pulmonary gas exchange during exercise in patients with chronic airway obstruction. Clin Sci 1966; 31:39-50.

11 Saunders NA, Powles ACP, Rebuck AS. Ear oximetry: accuracy and practicability in the assessment of arterial oxygenation. Am Rev Respir Dis 1976;113:745-9.

12 Powles ACP, Sutton JR, Wicks JR, Oldridge NB, Jones NL. Reduced heart rate response to exercise in ischemic heart disease: the fallacy of the target heart rate in exercise testing. Medical Science of Sports Exercise 1979;11:227-33.

13 Jones NL. Pathophysiology of hypoxia: protocol 2. Seminars in Respiratory Medicine 1981;3:118-24.

14 Cotes JE. Response to progressive exercise: a three index test. Br J Dis Chest 1972;66:160-84.

15 Spiro SG, Juniper E, Bowman P, Edwards RHT. An increasing work rate test for assessing the physiological strain of submaximal exercise. Clin Sci Molec Med 1974;46:191-206.

16 Wasserman K, Whipp BJ. Exercise physiology in health and disease. Am Rev Respir Dis 1975;112:219-49.

17 Clausen JL, ed. Pulmonary function testing: guidelines and controversies. New York: Academic Press, 1982.

is American Thoracic Society. Committee on guidelines for disability assessment. ATS News 1981;2.

19 American College of Sports Medicine. Guidelines for graded exercise testing and exercise prescription. 2nd ed. Philadelphia: Lea and Febiger, 1980.

20) Whipp BJ, Davis JA, Torres F, Wasserman K. A test to determine parameters of aerobic function during exercise. J Appl Physiol: Respiration, Environmental, and Exercise Physiology 1981;50:217-21.

${ }^{21}$ Rochmis P, Blackburn H. Exercise tests. A survey of pocedures, safety and litigation experience in approximately 170000 tests. JAMA 1971;217:1061-6.

22 Jones NL, Jones G, Edwards RHT. Exercise tolerance in chronic airway obstruction. Am Rev Respir Dis 1971; 103:477-91.

23 Wicks JR, Sutton JR, Oldridge NB, Jones NL. Comparison of the electrocardiographic changes induced by maximum exercise testing with treadmil and cycle ergometer. Circulation 1978;57:1066-70.

${ }^{24}$ Borg G, Noble B. Perceived exertion. In: Wilmore JH, ed. Exercise and sport sciences reviews. Vol. 2. New York: Academic Press, 1974:131-53. 